\title{
Perfil epidemiológico de pacientes portadores de Diabetes Mellitus tipo 2 residentes do município de Agudo, RS

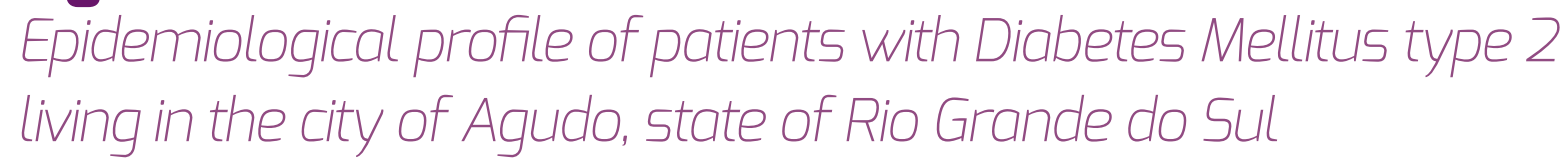

\author{
Laisa Dicow ${ }^{1}$ \\ Universidade de Santa Cruz do Sul (UNISC), Santa Cruz do Sul, RS, Brasil.
}

Recebido em: Junho 2015 / Aceito em: Dezembro 2015

laisadickow@yahoo.com.br

\section{RESUMO}

O Diabetes Mellitus (DM) é uma desordem metabólica, caracterizada por hiperglicemia crônica, com alterações do metabolismo, ocasionando importantes complicações irreversíveis. Objetivos: descrever o perfil epidemiológico dos pacientes que realizam os exames de glicemia em jejum e hemoglobina glicada em um laboratório do município de Agudo, RS, bem como comparar os níveis desses marcadores glicêmicos em pacientes atendidos pelo Sistema Único de Saúde (SUS) e por plano privado/particular. Método: realizou-se um estudo descritivo quantitativo retrospectivo, sendo os dados obtidos de janeiro a dezembro de 2013, através de prontuários digitalizados e acessados pelos programas Putty e Silab. Foram avaliados os seguintes parâmetros epidemiológicos: sexo, idade, tipo de atendimento (Sistema Único de Saúde - SUS ou planos privados/particulares), medicamentos utilizados e associação com outras doenças. Foram avaliados os valores de glicemia em jejum e hemoglobina glicada. Os dados foram analisados no programa SPSS 20,0. Foram realizadas estatísticas descritivas e univariadas. Resultados: dos 248 diabéticos estudados, $66,53 \%$ era do sexo feminino, $59,68 \%$ moradores da cidade e $78,63 \%$ atendidos pelo SUS, com idade média de 60 anos. Os valores médios de glicemia de jejum e hemoglobina glicada encontrados foram de $184,25 \mathrm{mg} / \mathrm{dL}$ e $8,60 \%$, respectivamente. Pacientes do SUS apresentaram média significativamente superior de glicemia de jejum $(p=0,038)$. Antidiabéticos e anti-hipertensivos foram os medicamentos mais utilizados pelos pacientes. Considerações finais: identificou-se que a maior parte dos pacientes pertencem ao sexo feminino e são atendidos pelo SUS. A média de glicose de jejum foi maior entre os pacientes atendidos pelo SUS, em comparação àqueles atendidos por planos privados/particulares.

Palavras-chave: Glicemia, Perfil de Saúde, Diabetes Mellitus.

\section{ABSTRACT}

Diabetes Mellitus (DM) is a metabolic disorder characterized by chronic hyperglycemia with metabolic disorders, leading to significant irreversible complications. Objectives: describe the epidemiological profile of patients with Type 2 Diabetes Mellitus (T2DM) checking the following parameters: gender, age, address, type of health insurance, medicines drugs, association with other diseases, fasting blood glucose value, glycated hemoglobin and compared the profile of patients with T2DM from government (SUS) and private health insurance plans. Method: we conducted a retrospective quantitative descriptive study, with data collected from January to December 2013, through digitalized records and accessed by Putty and Silab programs. The data were analyzed using SPSS 20.0 program. Descriptive and univariate statistics were performed. Results: Of the 248 diabetics studied, $66.53 \%$ were female, 59.68\% city dwellers and $78.63 \%$ from SUS. The average age was 60 and average value of fasting glucose and glycated hemoglobin, $184.25 \mathrm{mg} / \mathrm{dL}$ and $8.60 \%$, respectively. Antidiabetic and antihypertensive medications were the most cited medicines used by subjects. Final considerations: the majority of patients were female and SUS users. Fast glucose average was higher in SUS patients when comparing to those from private health insurance plans. Mellitus. 


\section{INTRODUÇÃO}

O Diabetes Mellitus (DM) é uma doença crônica, tendo por característica a elevação da glicose no sangue acima da taxa normal, juntamente com um conjunto de alterações metabólicas em decorrência da destruição das células beta do pâncreas, resistência à ação e/ou distúrbios da secreção da insulina, sendo causado por fatores genéticos e ambientais, conforme American Diabetes Association. ${ }^{1}$

Classifica-se, atualmente, o diabetes mellitus em 4 categorias: Diabetes Mellitus tipo 1; Diabete Mellitus tipo 2; outros tipos específicos de diabetes e diabetes gestacional. $^{2}$

O Diabetes Mellitus tipo 2 (DMT2) ou não insulino-dependente é uma doença heterogênea, em que ocorrem graus váriaveis de resistência à insulina, diminuição da secreção da insulina e do aumento da produção de glicose, sendo que na resistência à insulina ocorre à menor captação de glicose pelos tecidos periféricos em resposta à ação da insulina; assim, os tecidos adiposo e muscular não conseguem utilizar a glicose, prejudicando a supressão da lipólise, elevando os ácidos graxos livres e alterando mais ainda o transporte da glicose pelos músculos esqueléticos. No fígado, o aumento da glicose é compensado pelo aumento da secreção da insulina (na fase inicial), mas, após períodos prolongados, associa-se um efeito glicotóxico, ocorrendo a diminuição da função da célula beta do pâncreas. Para manter os níveis de glicose no sangue, o pancrêas tenta restabelecer o equilíbrio produzindo mais insulina, mas se permanecer descontrolado, as células tornam-se cada vez mais resistentes à insulina, mesmo o pancrêas produzindo mais este hormônio, ocasinando níveis elevados de insulina e esgotando o pâncreas. ${ }^{3-6}$

O diabetes está entre as 5 doenças que mais matam, chegando cada vez mais ao topo da lista. De 2010 a 2012, foi responsável por mais de 470 mil mortes em todo o Brasil. Neste período, o número saltou de 35,20 mil para 54,80 mil. Isso significa que a taxa de mortalidade avançou de 20,80 para 28,70 mortes por 100 mil habitantes, conforme o Ministério da Saúde. As cidades das regiões Sul e Sudeste, consideradas de maior desenvolvimento econômico, apresentaram as maiores prevalências desta patologia. ${ }^{7,8}$

Alguns fatores podem influenciar o surgimento do DMT2, como a obesidade, o sedentarismo, a maior taxa de urbanização, industrialização, o consumo de dietas hipercalóricas e de rápida absorção, mudança do estilo de vida, inatividade, o aumento da expectativa de vida, sendo que hoje a sobrevida de pessoas diabéticas é acima de 65 anos. . $^{9,10}$

Os sinais e sintomas do DMT2 evoluem lentamente, dificultando a descoberta e o estabelecimento de diagnóstico precoce. Assim, muitas pessoas recebem o diagnóstico após a manifestação de complicações da doença, como as cardiovasculares, insuficiência renal, amputação de pés e pernas, dentre outras, sendo que as doenças oculares, renais e vasculares são as maiores causas de invalidez e incapacitação para o trabalho..$^{10-13}$ Dessa forma, o Diabetes acomete populações, causando impacto social e econômico em todos os estágios de desenvolvimento. É uma doença que vem sendo reconhecido como problema de saúde pública com reflexos sociais importantes. ${ }^{10}$ Assim, o exame de glicemia jejum e hemoglobina glicada ( $\mathrm{HbA1c}$ ) funcionam como ferramenta de fundamental importância para avaliação do impacto do controle glicêmico sobre as complicações crônicas a longo prazo. ${ }^{8}$

Desta forma, o objetivo deste trabalho é descrever o perfil epidemiológico dos pacientes que realizam os exames de glicemia em jejum e hemoglobina glicada em um laboratório do município de Agudo, RS, bem como comparar os níveis desses marcadores glicêmicos em pacientes atendidos pelo Sistema Único de Saúde (SUS) e por plano privados/particulares.

\section{MÉTODO}

Foi realizado um estudo descritivo quantitativo retrospectivo referente ao perfil dos pacientes portadores de DMT2 que realizaram seus exames no posto de coleta de um laboratório no município de Agudo, RS. Somente este posto de coleta realiza atendimento vinculado ao Sistema Único de Saúde, em todo o município.

Foram analisados pacientes atendidos pelo Sistema Único de Saúde (SUS) e por planos privados/ particulares. As coletas de dados foram realizadas durante o período de janeiro a dezembro de 2013, através de dados cadastrais em prontuários digitalizados dos pacientes, sendo o acesso a estes dados através dos programas Putty (cadastro dos dados pessoais do paciente no momento do cadastro dos exames solicitados pelo médico) e o Silab (dados referentes aos exames e resultados dos exames de cada paciente).

Foi verificado, a cada mês, quem realizou o exame de glicose e posteriormente estes pacientes foram classificados conforme os valores de referência para este exame em: normal $(65,0$ a $99,0 \mathrm{mg} / \mathrm{dL})$; alterado (investigar; 100,0 a $125,0 \mathrm{mg} / \mathrm{dL}$ ) e sugere diabetes (confirmar; $\geq 126,0 \mathrm{mg} / \mathrm{dL}$ ). Todos os pacientes que apresentaram valor de glicemia $\geq 126 \mathrm{mg} / \mathrm{dL}$ entraram no grupo de estudo do presente trabalho. Após esta prévia classificação dos pacientes diabéticos, foram coletados os seguintes dados sobre cada indivíduo: sexo, idade, endereço (morador da cidade ou interior do município), tipo de atendimento (SUS ou planos privados/particulares), medicamentos utilizados, possíveis doenças relacionadas; também, foi avaliado o exame de hemoglobina glicada.

Todos os pacientes diabéticos que foram incluídos no presente estudo foram aqueles que realizaram o exame de glicose pela primeira vez, não sendo novamente contabilizados caso viessem a repetir o exame de glicose posteriormente, para assim, não influenciar no real número e perfil dos pacientes diabéticos. Todas as informações coletadas foram repassadas para uma planilha do Programa Microsoft Office Excel 2003 e, posteriormente, os dados analisados estatisticamente no programa SPSS 20,0 . Foram realizadas estatísticas descritivas e univariadas. Para a comparação dos indicadores demográficos e glicêmicos, de acordo com o tipo de atendimento, utilizou-se o teste exato de Fisher ou qui-quadrado. As variáveis contínuas foram analisadas pelo teste $t$ de Student.

O nome de cada paciente que participou desta pesquisa foi mantido sob sigilo. O presente estudo foi 
apreciado e aprovado pelo Comitê de Ética da Universidade de Santa Cruz do Sul, sob o número do CAAE 24603613.0.0000.5343.

\section{RESULTADOS}

A coleta dos dados dos portadores de DMT2 fo referente aos meses de janeiro a dezembro de 2013. Um total de 3223 pessoas realizaram o exame de glicose de jejum; destes, 248 apresentaram o valor de glicose jejum $\geq 126 \mathrm{mg} / \mathrm{dL}$, totalizando, assim, o grupo amostral do presente estudo, sendo esta população estudada classificada como "Sugere Diabetes".

A tabela 1 representa o perfil dos pacientes portadores de DMT2 que realizaram seus exames no Posto de Coleta. Dessa forma, foi possível observar, no presente estudo, uma prevalência de pessoas do sexo feminino $(66,53 \%)$, sendo a média de idade da população estudada de 60 anos, sendo a maior parte de moradores da cidade $(59,68 \%)$. A maioria dos diabéticos realizaram seus exames por intermédio do SUS (78,63\%). O valor médio de glicemia jejum e hemoglobina glicada foi de $184,25 \mathrm{mg} / \mathrm{dL}$ e $8,60 \%$, respectivamente. Os medicamentos mais utilizados pelos diabéticos estudados são os antidiabéticos orais $(25,63 \%)$, seguido dos anti-hipertensivos $(22,39 \%)$ e insulina, sendo menos representativa (2,25\%). A hipertensão arterial $(22,39 \%)$ e a dislipidemia $(9,15 \%)$ são as doenças associadas ao DMT2 mais encontradas.

$\mathrm{Na}$ tabela 2, há a representação da distribuição dos indivíduos que realizaram o exame de glicemia de jejum, durante o ano de 2013. Um total de 3223 pessoas compareceram ao laboratório para realizar o exame de glicemia de jejum; destes, identificou-se que $21,10 \%$ apresentaram alteração e 10,10\% sugere Diabetes. Ressaltamos que o grupo de estudo foi baseado em 248 diabéticos $(7,70 \%)$, sendo que alguns indivíduos repetiram o exame em algum outro mês do ano. Porém, para a análise do perfil epidemiológico, os indivíduos deste grupo foram contabilizados somente uma única vez, para assim não influenciarem no resultado final
Tabela 1 - Caracterização dos sujeitos. Agudo, RS, $2013(N=248)$

\begin{tabular}{lc}
\hline Características & $\mathbf{n}(\%)$ \\
\hline Sexo & $165(66,53)$ \\
Feminino & $83(33,47)$ \\
$\quad$ Masculino & $60,03( \pm 13,15)$ \\
Idade (anos)* & \\
Endereço & $148(59,68)$ \\
$\quad$ Cidade & $100(40,32)$ \\
Interior & \\
Convênio & $195(78,63)$ \\
SUS & $53(21,37)$ \\
Particular/Plano de Saúde & $184,25( \pm 65,31)$ \\
Glicose de jejum (mg/dL)* & $8,60( \pm 2,10)$ \\
Hemoglobina glicada (\%)* & \\
Medicamentos mais citados**(N=710) & $182(25,63)$ \\
Antidiabéticos Orais & $159(22,39)$ \\
Anti-hipertensivos & $139(19,58)$ \\
Outros & $65(9,15)$ \\
Dislipemicos & $50(7,04)$ \\
Diuréticos & $48(6,76)$ \\
Antiinflamatórios & $27(3,80)$ \\
Antiulcerosos & $16(2,25)$ \\
Insulina & $13(1,83)$ \\
Sedativos & $11(1,55)$ \\
Não tomam medicamentos &
\end{tabular}

*Valores expressos em média (desvio-padrão); * *questão de múltipla escolha.

do real perfil dos diabéticos. Destaca-se, ainda, que os meses de maio e outubro foram os que apresentaram maior número de pessoas que realizaram seus exames (328 e 311 pessoas, respectivamente).

O presente estudo comparou o perfil sociodemográfico dos portadores DMT2 que realizam seus exames por intermédio do SUS ou planos de saúde/particulares, a fim de identificar se houve alguma diferença entre estes dois grupos. Os dados estão representados na tabela 3. Não foram observadas diferenças significativas na comparação das variáveis sociodemográficas, de acordo com o tipo de atendimento. Por outro lado, observou-se que a média de glicose foi significativamente superior entre os indivíduos atendidos pelo SUS $(p=0,038)$.

Tabela 2 - Exames do perfil glicêmico realizados no laboratório. Agudo, RS, 2013.

\begin{tabular}{|c|c|c|c|c|}
\hline Mês/2013 & Normal n (\%) & Alterado n (\%) & Sugere Diabetes n (\%) & Total n (\%) \\
\hline Janeiro & $180(73,10)$ & $40(16,30)$ & $26(10,60)$ & 246 \\
\hline Fevereiro & $185(69,50)$ & $53(20,00)$ & $28(10,50)$ & 266 \\
\hline Março & $188(74,30)$ & $48(19,00)$ & $17(6,70)$ & 253 \\
\hline Abril & $174(68,00)$ & $58(22,50)$ & $25(9,50)$ & 254 \\
\hline Maio & $232(70,70)$ & $64(19,50)$ & $32(9,80)$ & 328 \\
\hline Junho & $165(68,20)$ & $52(21,50)$ & $25(10,30)$ & 242 \\
\hline Julho & $195(71,00)$ & $55(20,00)$ & $25(9,00)$ & 275 \\
\hline Agosto & $169(62,60)$ & $68(25,20)$ & $33(12,20)$ & 270 \\
\hline Setembro & $203(79,40)$ & $29(11,60)$ & $23(9,00)$ & 255 \\
\hline Outubro & $195(62,70)$ & $79(25,40)$ & $37(11,90)$ & 311 \\
\hline Novembro & $183(65,10)$ & $70(24,90)$ & $28(10,00)$ & 281 \\
\hline Dezembro & $149(61,60)$ & $64(26,60)$ & $28(11,80)$ & 242 \\
\hline
\end{tabular}


Tabela 3 - Exames do perfil glicêmico realizados no laboratório. Agudo, RS, 2013.

\begin{tabular}{|c|c|c|c|}
\hline & \multicolumn{2}{|c|}{ TIPO DE ATENDIMENTO } & \multirow[b]{2}{*}{$p$} \\
\hline & sus & Particular/plano de saúde & \\
\hline \multicolumn{4}{|l|}{ Sexo } \\
\hline Feminino & $130(78,80)$ & $35(21,20)$ & 1,000 \\
\hline Masculino & $65(78,30)$ & $18(21,70)$ & \\
\hline Idade (anos) $^{*}$ & $58,42( \pm 12,43)$ & $65,98( \pm 14,13)$ & 0,001 \\
\hline \multicolumn{4}{|l|}{ Endereço } \\
\hline Cidade & $114(77,00)$ & $34(23,00)$ & 0,529 \\
\hline Interior & $81(81,00)$ & $19(19,00)$ & \\
\hline \multicolumn{4}{|l|}{ Faixa etária } \\
\hline $17-40$ anos & $16(88,90)$ & $2(11,10)$ & 0,009 \\
\hline 41-61 anos & $93(86,10)$ & $15(13,90)$ & \\
\hline$\geq 62$ anos & $86(70,50)$ & $36(29,50)$ & \\
\hline Glicose de jejum $(\mathrm{mg} / \mathrm{dL})^{*}$ & $188,72( \pm 68,28)$ & $167,79( \pm 50,18)$ & 0,038 \\
\hline Hemoglobina glicada $(\%) *$ & $8,3( \pm 56,87)$ & $9,2( \pm 75,65)$ & 0,268 \\
\hline
\end{tabular}

\section{DISCUSSÃO}

Como no presente estudo, outros trabalhos também identificaram que a maioria dos sujeitos avaliados são do sexo feminino, como podemos citar os trabalhos realizados em Ribeirão Preto $(79,80 \%),{ }^{10}$ Florianópolis $(60,00 \%),{ }^{17}$ Macapá $(66,20 \%),{ }^{14}$ Joaçaba $(66,70 \%)^{15} \mathrm{e}$ Porto Alegre $(67,20 \%) .{ }^{16}$ É interessante citar que alguns autores justificam a maior presença feminina entre os estudos envolvendo DMT2, devido ao fato de que as mesmas preocupam-se mais com a saúde, procuram mais os serviços de atendimento à saúde e, consequentemente, têm mais acesso ao diagnóstico da doença, sendo que muitas vezes, o homem é o provedor da família, o que dificulta a procura destes por serviços de saúde. Outro aspecto relevante é o envelhecimento da população; assim, alguns autores ${ }^{10,14-18}$ citam que uma maior parcela da população vem envelhecendo em maior número e de forma rápida, havendo, assim, um aumento do número de mulheres com mais de 60 anos, que hoje representam $55,10 \%$ da população idosa. Fato que pode ser observado no presente estudo, quando comparado ao estudo realizado em Porto Alegre, que estudou 125 diabéticos, identificando maior prevalência de pessoas do sexo feminino na faixa etária de 60 a 69 anos de idade. ${ }^{16}$ Considerando que o DMT2 é característico da idade adulta, a incidência é progressivamente maior com o processo de envelhecimento, aumentando particularmente após os 40 anos, em que a média de idade dos pacientes é de 60 anos. O DMT2 é o mais prevalente, correspondendo a cerca de $90 \%$ dos casos de diabetes. ${ }^{10,19}$

Em relação ao local de moradia dos portadores de DMT2 estudados, observou-se que a maioria dos diabéticos são residentes da cidade, devido ao acesso ser facilitado e rápido em relação aos moradores do interior do município. Muitos dos moradores do interior precisam percorrer uma considerável distância até a cidade e, consequentemente, até o posto de coleta, pois muitos dos diabéticos estudados residem em localidades rurais bem distantes da cidade, podendo ressaltar o fato que muitos destes indivíduos vão para a cidade somente em momentos realmente necessários.

Indivíduos pré-diabéticos podem apresentar níveis de glicose acima do normal, mas não elevados o sufi- ciente para o diagnóstico de DM, sendo assintomáticos, permanecendo neste circuito vicioso não reversível, que inclui efeitos deletérios graves sobre o metabolismo da glicose. Assim, é de extrema importância realizar o exame de glicemia de jejum frequentemente, juntamente com o exame de hemoglobina glicada, os quais fornecem valores da taxa de glicose no sangue, no momento da coleta e 120 dias anteriores à coleta de sangue, respectivamente..$^{3,19-21}$

Como observado no presente estudo, o trabalho realizado em Ribeirão Preto também obteve resultado alterado $(9,30 \%)$ para o valor médio de $\mathrm{HbA} 1 \mathrm{c}$, sendo este exame indicado para acompanhar os níveis glicêmicos ao longo do tempo, pois possibilita medir o valor do pico nos últimos 120 dias, não sofrendo interferência nem flutuações como a medida de glicemia jejum. As determinações periódicas da hemoglobina glicada são de grande utilidade no acompanhamento dos diabéticos, visando a obtenção de um controle adequado. ${ }^{18}$

Identificou-se, no presente estudo, que os antidiabéticos orais e anti-hipertensivos são os medicamentos mais utilizados. Outros estudos também identificaram a utilização de medicamentos pelos participantes, sendo o antidiabético oral o mais utilizado e o menos utilizado a insulina, no estudo realizado em Macapá. ${ }^{14}$ No trabaIho em Ribeirão Preto, $64,20 \%$ dos participantes utilizavam uma associação de insulina e antidiabéticos orais e $35,80 \%$ utilizavam a insulina. ${ }^{10}$ No trabalho em Porto Alegre, $54,40 \%$ utilizavam hipoglicemiantes orais, $12 \%$ utilizavam, além da medicação oral, a insulina e 8,80\% utilizavam apenas insulina para o tratamento de DMT2. ${ }^{16}$ Destaca-se que a correta ingestão dos antidiabéticos é a principal estratégia para o alcance da normoglicemia. ${ }^{19}$

Hipertensão arterial e dislipidemia foram as doenças associadas mais encontradas no presente estudo; estas também foram as mais representativas no estudo realizado em Ribeirão Preto, com $87,70 \%$ e 72,80\%, respectivamente. ${ }^{18}$ Estudo realizado em Porto Alegre identificou que $76,80 \%$ dos participantes apresentavam hipertensão e $48 \%$ dos diabéticos apresentavam dislipidemia associada. ${ }^{16}$ Existe um consenso entre os autores, que a hipertensão é cerca de duas vezes mais frequente entre indivíduos diabéticos, quando comparados à população em geral, estando presente em $50 \%$ nos pacientes no momento do diagnóstico de DMT2. 
No estudo de Joaçaba, 30\% dos participantes eram portadores somente de DMT2 e 70\% eram portadores de DMT2 e hipertensão arterial. ${ }^{15} \mathrm{O}$ DMT2 é um distúrbio crônico, complexo e de difícil tratamento ao longo do tempo; de modo geral, a maior parte desses sujeitos está hipertenso e com excesso de peso, impossibilitando que permaneçam sem o uso de antidiabéticos orais. O não cumprimento do regime medicamentoso desfavorece benefícios fisiológicos esperados (normoglicemia) podendo ocasionar complicações, principalmente micro e macrovasculares, como infarto agudo do miocárdio, acidente vascular encefálico, insuficiência renal, retinopatias, amputações e óbitos diretos. Atualmente, o tratamento para manter a normoglicemia é a adoção de dieta hipocalórica, aumento da prática de exercícios físicos e uso de medicações (quando necessário), pois diminuindo a ingestão de alimentos calóricos já se reduz três dos fatores de risco para doença cardiovascular, que são a obesidade, a dislipidemia e a hipertensão arterial. ${ }^{16,22,23}$

Houve certa linealidade na distribuição de pessoas que realizaram o exame de glicemia de jejum durante o ano. Mas, nos meses de maio e outubro, houve uma parcela maior de indivíduos, sugerindo que as variações térmicas podem ter influenciado, aumentando a procura das pessoas, tanto para atendimento médico, como para realização de exames. O mês de outubro destacou-se devido a periodicidade na realização dos exames. As es tações do ano são bem definidas no Rio Grande do Sul, interferindo no hábito social, físico e alimentar, sendo que na estação fria, todos os hábitos tendem a se alterar; dessa forma, as pessoas ingerem uma quantidade maior de alimentos que contêm carboidratos e lipídios. Alterações alimentares, associadas a mudanças demográficas e sociais, vem sendo observadas em diversos países do mundo, acarretando no aumento das taxas de sobrepeso e obesidade, sendo os principais fatores que explicam o crescimento da prevalência do DMT2. ${ }^{24}$

Quando se comparou o grupo que realizou seus exames pelo SUS e por intermédio de planos de saúde/particular, novamente houve prevalência do sexo feminino, igualmente observado no estudo realizado em Porto Alegre, ${ }^{16}$ em que $67,20 \%$ da população era feminina, na faixa etária de 60 a 69 anos de idade, fato também observado em diversos outros trabalhos. ${ }^{10,14,17}$

Em relação à idade média dos grupos, no estudo de Joaçaba, a média de idade de surgimento da DMT2 foi aos 57,63 anos de idade $( \pm 10,63)$, sendo a faixa de idade acima dos 40 anos em que se relata comumente a fase de início da DMT2. O predomínio desta faixa etária (41-61 anos) também pode ser observado no trabalho de Macapá, em que 51,50\% dos 68 diabéticos se enquadravam na faixa etária de 40 a 60 anos, sendo seguido da faixa etária $\geq 60$ anos, com 39,70\% dos indivíduos. ${ }^{14} \mathrm{O}$ trabalho em Ribeirão Preto também identificou resultados similares, em que $83,80 \%$ entram na faixa etária de 30 e 50 anos. A elevação da glicemia com a idade é fato reconhecido pela literatura mundial e o DMT2 é certamente a doença mais comum entre os idosos, sendo que muitos desses pacientes foram diagnosticados aos 40-50 anos e envelheceram com a enfermidade. ${ }^{10} \mathrm{O}$ estudo realizado na população mexicana ${ }^{25}$ observou que uma porção significativa de indivíduos tem uma apresentação clínica de DMT2 em idade mais jovem ( $25 \%$ da população). Já, a população que realiza seus exames por meio de planos de saúde/ particular são as de maior faixa etária, pois esta já está normalmente conveniada a algum plano de saúde, necessitando buscar atendimento médico mais frequentemente, até para solucionar ou controlar outras possíveis doenças. ${ }^{15,19,25,26}$

Sugere-se que esta diferença na faixa etária dos grupos do presente estudo seja devido a uma parcela cada vez mais jovem da população estar preocupada com sua saúde, buscando atendimento médico, bem como os médicos terem atenção para esta fase da vida, solicitando exames para identificar o DM. Sugere-se, também, que o SUS tenha uma maior representação nesta faixa etária devido aos indivíduos terem o atendimento médico e a realização dos exames sem custo algum, tornando-se atrativo para aquelas pessoas que nesta faixa etária está inserida no mercado de trabalho e que são mantenedores de suas famílias, facilitando o acesso à assistência médica sem grandes custos. 0 estudo em Joaçaba identificou que $70 \%$ dos entrevistados descobriram a patologia por meio de exames de rotina, ressaltando assim a importância de realizarem-se estes exames a partir dos 40 anos, idade de surgimento da DMT2, evitando assim futuras complicações da doença. ${ }^{15}$

No estudo realizado no México, ${ }^{25}$ a incidência de DMT2 foi maior em indivíduos de baixa renda em comparação com os de alta renda, sendo a pobreza um efeito promotor, efeito este também observado no presente estudo. A maioria das pessoas que procuram assistência médica no Posto de Saúde do município de Agudo são de baixa renda; assim, fatores como baixo nível socioeconômico e escolaridade têm sido associados a uma menor adesão ao tratamento e maior morbidade relacionada ao DM, sendo que estes fatores influenciam diretamente no padrão de vida das pessoas, havendo diferença na alimentação (refletindo na normalidade glicemica), na prática de exercícios físicos, correta ingestão dos medicamentos, cuidados com possíveis complicações, sendo que a influência deste meio externo muitas vezes se sobrepõe até as instruções médicas. ${ }^{19,25,27}$

Quando se comparou a média de glicose de jejum, se observou uma diferença entre os grupos $(p=0,038)$, em que a maior média pertenceu ao grupo do SUS, demonstrando que os pacientes, além de apresentarem uma faixa etária menor, estão com os níveis de glicemia de jejum mais altos, demonstrando um descontrole da glicemia, identificando assim o surgimento da doença cada vez mais cedo. Para o valor médio de $\mathrm{HbA} 1 \mathrm{c}$, não houve diferença significativa. A dosagem de HbA1c é indicada para DMT2, para efeito de controle, em que a meta a se atingir é o valor abaixo de $7 \%$, tanto para adultos, como adultos jovens; já, para idosos, o nível deve ser abaixo de $8 \%$. Valores acima de $7 \%$ estão associados a um risco progressivamente maior de complicações crônicas. Estudos demonstram esta associação linear entre os níveis de HbA1c e complicações micro e macrovasculares, sendo que quanto maior os níveis de glicose no sangue ao longo do tempo, maior a associação com o desenvolvimento de alterações associadas 
com o DM, sendo este exame fundamental e determinante para o acompanhamento dos diabéticos. ${ }^{28,29}$

\section{CONSIDERACÕ̃ES FINAIS}

O presente estudo identificou que a maior parte dos pacientes pertencem ao sexo feminino e são atendidos pelo SUS. Antidiabéticos orais e anti-hipertensivos foram os principais medicamentos utilizados pelos pacientes. A média de glicose de jejum foi maior entre os pacientes atendidos pelo SUS, em comparação àqueles atendidos por planos privados/particulares.

\section{REFERÊNCIAS}

1. Marcelino DB, Carvalho MDB. Reflexões sobre o diabetes tipo 1 e sua relação com o emocional. Psicol reflex crit 2005;18(1):72-7.

2. Gross JL, Silveiro SP, Camargo JL, Reichelt AJ, Azavedo MJ. Diabetes melito: diagnóstico, classificação e avaliação do controle glicêmico. Arq bras endocrinol metab. 2002 Fev;46(1).

3. Boada CAC, Martínez-Moreno JM. Pathophysiology of diabetes mellitus type 2: beyond the duo "insulin resistancesecretion deficit". Nutr hosp 2013;28(2):78-87.

4. Gabbay M, Cesarini PR, Dib SA. Diabetes melito do tipo 2 na infância e adolescência: revisão de literatura. J pediatr 2003;79(3):201-8.

5. Gill A, Kukreja S, Malhotra N, Chhabra N. Correlation of the serum insulin and serum uric acid levels with the glycated haemoglobin levels in the patients of type 2 diabetes mellitus. Journal of clinical diagnostic research 2013;7(7):1295-7.

6. Soliman GZA. Blood lipid peroxidation (superoxide dismutase, malondialdehyde, glutathione) levels in Egyptian type 2 diabetic patients. Singap med j 2008;49(2):129-136.

7. Ministério da Saúde. Brasil. Portal da Saúde. Diabetes Mellitus; [citado em 2014 Jul 01]. Disponível em: <http:// portalsaude.saude.gov.br>.

8. Sumita NM, Andriolo A. Importância da determinação da hemoglobina glicada no monitoramento do paciente portador de diabetes mellitus. J bras patol med lab 2006;42(3).

9. Arsa G, Lima L, Almeida SS, Moreira SR, Campbell CSG, Simões HG. Diabetes mellitus tipo 2: aspectos fisiológicos, genéticos e formas de exercício físico para seu controle. Rev bras cineantropom desempenho hum 2009;11(1):103-11.

10. Ortiz MCA, Zanetti ML. Levantamento dos fatores de risco para diabetes mellitus tipo 2 em uma instituição de ensino superior. Rev Latino-am Enferm 2001;9(3):58-63.

11. Alvim RO, Santos PCJL, Musso MM, Cunha RS, Krieger JE, Mill JG, Pereira AC. Impact of diabetes mellitus on arterial stiffness in a representative sample of an urban Brazilian population. Diabetol metab syndr 2013;5(45).

12. Barbosa RB, Barceló A, Machado CA. Campanha nacional de detecção de casos suspeitos de diabetes mellitus no Brasil: relatório preliminar. Rev Panam Salud Publica/Pan Am J Public Health 2001;10(5).

13. Tavares DMS, Rodrigues RAP. Educação conscientizadora do idoso diabético: uma proposta de intervenção do enfermeiro.
Rev Esc Enferm 2002;36(1):88-96.

14. Almeida ANF, Cárdenas AMC. Caracterização epidemiológica de pacientes com diabetes mellitus tipo 2 de uma unidade básica de saúde de Macapá. Revista de ciências da Amazônia 2013;1(1):74.

15. Bonetti E, Schaly D, Rover C, Fiedler MM. Atividade física em indivíduos portadores de diabetes mellitus do município de Joaçaba. Evidência - Ciência e Biotecnologia 2012;12(1):41-50.

16. Grillo MFF, Gorini MIPC. Caracterização de pessoas com diabetes mellitus tipo 2. Rev Bras Enferm Bras 2007;60(1):49-54.

17. Tavares BC, Barreto FA, Lodetti ML, Silva DMGV, Lessmann JC. Resiliência de pessoas com diabetes mellitus. Florianópolis (SC). Texto \& contexto enferm 2011;20(4):751-7.

18. Villas Boas LCG, Foss-Freitas MC, Pace AE. Adesão de pessoas com diabetes mellitus tipo 2 ao tratamento medicamentoso. Rev bras enferm 2014;67(2):268-73.

19. Almeida ANF. Qualidade de vida de pacientes com Diabetes Mellitus: Estudo comparativo de dois programas assistenciais da unidade básica de saúde da Universidade Federal do Amapá, no município de Macapá, Amapá. [Dissertação de Mestrado]. Amapá: Universidade Federal do Amapá, Programa de Pós-Graduação em Ciências da Saúde; 2012.

20. Ferreira AF. Hemoglobina glicada no diagnóstico, monitoramento de pacientes diabéticos e o uso de modelos didáticos na prática da docência. Curso de Ciências Biológicas, Centro Universitário Metodista Izabela Hendrix.

21. Paiva C. Novos critérios de diagnóstico e classificação da diabetes mellitus. Med intern 2001;7(4).

22. Araújo LMB, Britto MMS, Cruz TRP. Tratamento do diabetes mellitus do tipo 2: novas opções. Arq Bras Endocrinol Metab 2000;44(6).

23. Araújo MFM, Freitas RWJF, Fragoso LVC, Araújo TM, Damasceno MMC, Zanetti ML. Cumprimento da terapia com antidiabéticos em usuários da atenção primária. Florianópolis (SC). Texto \& contexto enferm 2011;20(1):135-43.

24. Benetti F, CENI GC. Frequencia do consumo alimentar de pacientes diabéticos em acompanhamento ambulatorial na região norte do $\mathrm{RS}$, do segundo semestre de 2008. Vivências 2010;6(9):124-35.

25. González-Villalpando C, Dávila-Cervantes CA, ZamoraMacorra M, Trejo-Valdivia B, González-Villalpango ME. Incidence of type 2 diabetes in Mexico. Results of the Mexico city diabetes study after 18 years of follow-up. Salud pública Méx 2014;56(1):11-17.

26. Silva ALS, Karino ME, Mattos ED, Campos EC, Spagnuolo RS. Perfil epidemiológico dos idosos de uma unidade saúde da família. Ciênc Biol Saúde 2009;11(2):27-33.

27. Duarte AA. Representação social e coping: uma forma de elaboração da diabetes tipo 2.Brasília: Centro Universitário de Brasília, Curso de Psicologia.

28. Bem AF, Kunke J. A importância da determinação da hemoglobina glicada no monitoramento das complicações crônicas do diabetes mellitus. J Bras Patol Med Lab 2006;42(3):185-91.

29. Braga JR, Avezum A, Ferreira SRG, Forti A. Management of diabetes mellitus and associated cardiovascular risk factors in Brazil - the Brazilian study on the practice of diabetes care. Diabetol metab syndr 2013;46(5). 